\title{
Patient perspectives on biological treatments for inflammatory arthritis: A multi-center study in Korea
}

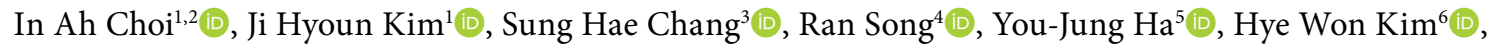

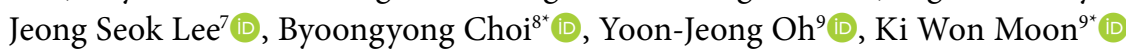 \\ * These authors contributed equally.
}

${ }^{1}$ Department of Internal Medicine, Division of Rheumatology, Chungbuk National University Hospital, Cheongju, South Korea

${ }^{2}$ Department of Internal Medicine, College of Medicine, Chungbuk National University, Cheongu, South Korea

${ }^{3}$ Department of Internal Medicine, Division of Rheumatology, Soonchunhyang University Hospital, Cheonan, South Korea

${ }^{4}$ Department of Internal Medicine, Division of Rheumatology, Kyung Hee University, Seoul, South Korea

${ }^{5}$ Department of Internal Medicine, Division of Rheumatology, Seoul National University Bundang Hospital, Seongnam, South Korea

${ }^{6}$ Department of Internal Medicine, Hospital Medicine Center, Seoul National University Bundang Hospital, Seongnam, South Korea

${ }^{7}$ Korea Advanced Institute of Science and Technology, Graduate School of Medical Science and Engineering, Daejeon, South Korea

${ }^{8}$ Department of Internal Medicine, Division of Rheumatology, Seoul Metropolitan Seoul Medical Center, Seoul, South Korea

${ }^{9}$ Department of Internal Medicine, Division of Rheumatology, Kangwon National University School of Medicine, Chuncheon, South Korea

\begin{abstract}
Objectives: The aim of this study was to evaluate the patient's perception of the use of biological disease-modifying anti-rheumatic drugs (bDMARDs) and provide a basis for physicians to understand the patient's perspective.

Patients and methods: Between December 2018 and June 2019, a total of 307 patients (162 males, 145 females; median age: 48 years; range, 18 to 81 years) were included in this investigator-initiated, multi-center, observational, and cross-sectional study in six rheumatology centers. We asked patients using bDMARDs to treat rheumatoid arthritis (RA) or ankylosing spondylitis (AS) to complete a questionnaire regarding major considerations and satisfaction with bDMARDs, preferred administration route, knowledge about bDMARDs, experiences of adverse events, non-adherence, and expectations of their healthcare provider. The satisfaction of physician and clinical information on the patient's disease and treatment were also collected.

Results: Of the patients, 139 had RA and 168 had AS. Median disease duration was six years in RA and five years in AS. A total of $80.1 \%$ of the patients and $77.1 \%$ of the physicians indicated being satisfied or very satisfied with the therapeutic effect of the current bDMARD. Most patients were open to intravenous or subcutaneous injection, with the most preferred route of administration being subcutaneous (41.3\%), followed by intravenous $(32.0 \%)$, and oral $(26.7 \%)$. The patients considered therapeutic effect to be more important than cost or convenience while choosing a bDMARD (69.3\%), and most were willing to be educated about therapeutic effects (46.1\%). Only $35.2 \%$ of the patients reported well and/or very well knowledge about the therapeutic effects, side effects, and administration methods of their current bDMARD, and $86.6 \%$ cited their physician as the primary source of information about biological treatment.

Conclusion: Patients value therapeutic effect more than cost or convenience while selecting a bDMARD, and consider their physicians to be the primary information source. Therefore, it is important for physicians to provide appropriate education and encourage patients to cooperate actively with treatment.
\end{abstract}

Keywords: Ankylosing spondylitis, biological agent, Korea, patient education, rheumatoid arthritis.

Received: October 19, 2020 Accepted: December 11, 2020 Published online: June 24, 2021

Correspondence: Ki Won Moon, MD, PhD. Department of Internal Medicine, Division of Rheumatology, Kangwon National University School of Medicine, 24289, Chuncheon, South Korea. Tel: +82-33-258-2000 e-mail: kiwonmoon@kangwon.ac.kr

\section{Citation:}

Choi IA, Kim JH, Chang SH, Song R, Ha YJ, Won Kim, H, et al. Patient perspectives on biological treatments for inflammatory arthritis: A multi-center study in Korea. Arch Rheumatol 2021;36(4):499-509.

(02021 Turkish League Against Rheumatism. All rights reserved.

This is an open access article under the terms of the Creative Commons Attribution-NonCommercial License, which permits use, distribution and reproduction in any medium, provided the original work is properly cited and is not used for commercial purposes (http://creativecommons.org/licenses/by-nc/4.0/). 
Biological disease modifying anti-rheumatic drugs (bDMARDs) or biological agents are antibodies or neutralizing receptors produced using genetic recombination technology, and are used in treatment by blocking the function of substances or cells thought to be involved in the pathogenesis of inflammatory rheumatic diseases. ${ }^{1}$ Although these biological agents can cause discomfort at the injection site, have higher costs, and have an increased risk of infection compared to conventional disease modifying anti-rheumatic drugs (DMARDs), they have been proven efficacy in patients with inflammatory arthritis who are refractory to conventional DMARDs. ${ }^{2}$ For appropriate management of chronic inflammatory arthritis, such as rheumatoid arthritis (RA) and ankylosing spondylitis (AS), it is important that patients share their current status and discuss therapeutic options with their physicians. The European League Against Rheumatism (EULAR) recommendations for the management and treatment of RA and AS emphasize the shared decision-making process between patient and physician as an overarching principle. ${ }^{3,4}$ To make this shared decision-making process efficient, education should be provided to patients, and physicians should understand patients' thoughts, perceptions, and concerns regarding their treatment. There have been some studies about perception and preference of patients who use bDMARD; however, the spectrum of enrolled patients was limited, or main topic was focused on specific issue such as satisfaction, preference or adherence..$^{5-8}$ It is necessary to understand overall perception, thoughts or concern of patients who use bDMARD to establish appropriate treatment plans for various patients.

In the present study, we aimed to evaluate the patients' perception of the use of bDMARDs and provide a basis for physicians to understand the patient's perspective and concerns.

\section{PATIENTS AND METHODS}

This investigator-initiated, multi-center, observational, and cross-sectional study was conducted at six rheumatology centers in five cities of Korea (Cheongju, Cheonan, Seoul, Seongnam, and Chuncheon) between December 2018 and June 2019. A total of 307 patients
(162 males, 145 females; median age: 48 years; range, 18 to 81 years) receiving bDMARD for more than six months were included. A written informed consent was obtained each patient. The study protocol was approved by the Institutional Review Board of each study center. The study was conducted in accordance with the principles of the Declaration of Helsinki.

We asked participants to complete a patient questionnaire on using bDMARDs currently available for the treatment of RA or AS (i.e., etanercept, infliximab, adalimumab, golimumab, tocilizumab, abatacept, rituximab, and secukinumab). Only patients who used bDMARD for more than six months were included in this study. The questionnaire consisted of items regarding patients' expectations, major considerations and satisfaction with bDMARDs, preferred administration route, knowledge about the current using bDMARDs, experiences of adverse events, non-adherence, and expectations of their healthcare provider. The researchers who participated in this study developed a questionnaire, and it was verified that the meaning of each question was communicated well to four ( $n=2$ RA and $n=2$ AS) patients. Most questions were answered using a Likert-type scale ranging from 1 to 5 . An English translation of the patient questionnaire can be found in Supplement 1 . The case report form (CRF) for physicians consisted of patients' clinical information (sex, age, type of disease, and disease duration), patients' treatment (current use of bDMARD, duration of current bDMARD, number of previous bDMARDs used, duration of overall bDMARD use, and experience of adverse events), physician global assessment, disease activity, including Disease Activity Score-28 (DAS28) and its components (erythrocyte sedimentation rate $[\mathrm{ESR}]$, C-reactive protein [CRP], swollen joint counts among 28 joints, tender joint counts among 28 joints, and patient global assessment) for RA, ${ }^{9}$ Ankylosing Spondylitis Disease Activity Score (ASDAS) and its components (back pain, peripheral pain/swelling, duration morning stiffness, patient global assessment, ESR, CRP) for $A S,{ }^{10}$ and radiographic findings (radiographic erosion in RA, modified New York criteria in AS). Additionally, patients and physicians recorded their level of satisfaction with the current bDMARD on a Likert-type scale ranging from 1-5. 


\section{Statistical analysis}

The responses on the Likert-type scale were expressed as a distribution of the proportions of each five-stage response. The agreement between physicians' and patients' response was calculated using the weighted Cohen's kappa (к). The kappa results were interpreted as follows: values $\leq 0$ as no agreement, $0.01-0.20$ as none to slight, $0.21-0.40$ as fair, $0.41-0.60$ as moderate, $0.61-0.80$ as substantial, and $0.81-1.00$ as almost perfect agreement.

Statistical analysis was performed using the MedCalc version 18.6 software (MedCalc Software Bvba, Ostend, Belgium). The graphs were visualized using the GraphPad Prism for Windows version 7.00 (GraphPad Software Inc., CA, USA). Descriptive data were expressed in mean \pm standard deviation (SD), median (minmax) or number and frequency, where applicable. A $p$ value of $<0.05$ was considered statistically significant.

\section{RESULTS}

We collected data from 307 patients at six different hospitals in South Korea (Figure 1). Of the patients, 139 had RA and 168 had AS. Median disease duration was six years in RA and five years in AS. Table 1 shows demographic and clinical characteristics of the participants.

According to their satisfaction with the therapeutic effect of bDMARDs on a fivepoint Likert-type scale, $80.1 \%$ of the patients and $77.1 \%$ of the physicians answered as satisfied and/or very satisfied with their current treatment (Figure 2). There was also a substantial degree of correlation between the patients' and physicians' levels of satisfaction (weighted $\kappa: 0.67$, 95\% confidence interval $[\mathrm{CI}]$ : 0.59-0.74). Among RA patients, $77.7 \%$ of the patients and $76.8 \%$ of the physicians responded as satisfied and/or very satisfied with their current treatment (weighted $\kappa: 0.72$,

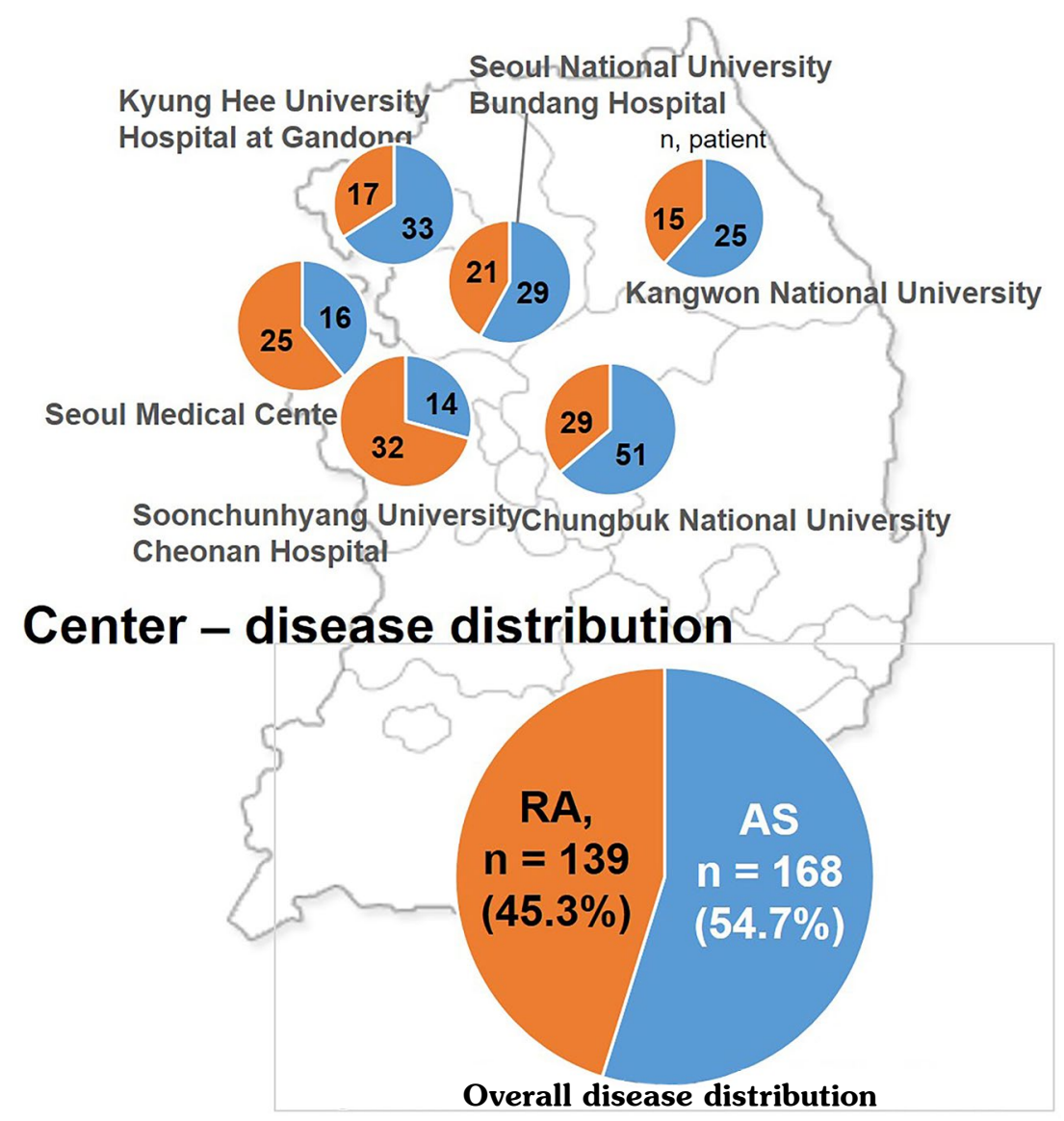

Figure 1. Study participant $(n=307)$ diagnosis and location. 
Table 1. Demographic and clinical characteristics of participants

\begin{tabular}{|c|c|c|c|c|c|c|c|c|c|c|c|c|}
\hline & \multicolumn{4}{|c|}{ RA (n=139) } & \multicolumn{4}{|c|}{ AS $(n=168)$} & \multicolumn{4}{|c|}{ Overall $(n=307)$} \\
\hline & $\mathrm{n}$ & $\%$ & Median & Range & $\mathrm{n}$ & $\%$ & Median & Range & $\mathrm{n}$ & $\%$ & Median & Range \\
\hline Age (year) & & & 58 & $22-81$ & & & 42 & $18-73$ & & & 48 & $18-81$ \\
\hline \multicolumn{13}{|l|}{ Sex } \\
\hline Male & 27 & 19.4 & & & 135 & 80.4 & & & 162 & 52.8 & & \\
\hline Disease duration (year) & & & 6 & $0-36$ & & & 5 & $0-40$ & & & 6 & $0-40$ \\
\hline Less than 2 years & 23 & 16.5 & & & 27 & 16.1 & & & 50 & 16.3 & & \\
\hline $2-5$ years & 45 & 32.4 & & & 50 & 29.8 & & & 95 & 30.9 & & \\
\hline More than 5 years & 69 & 49.6 & & & 89 & 53.0 & & & 158 & 51.5 & & \\
\hline \multicolumn{13}{|l|}{ Duration of biologic therapy } \\
\hline Less than 1 year & 43 & 30.9 & & & 42 & 25 & & & 85 & 27.6 & & \\
\hline $1-5$ years & 76 & 54.7 & & & 83 & 49.4 & & & 159 & 51.8 & & \\
\hline More than 5 years & 20 & 14.4 & & & 43 & 25.6 & & & 63 & 20.5 & & \\
\hline \multicolumn{13}{|c|}{ Number of previous biologic agents } \\
\hline 0 & 107 & 77.0 & & & 144 & 85.7 & & & 251 & 81.8 & & \\
\hline 1 & 18 & 12.9 & & & 22 & 13.1 & & & 40 & 13.0 & & \\
\hline 2 & 8 & 5.8 & & & 1 & 0.6 & & & 9 & 2.9 & & \\
\hline 3 or more & 6 & 4.3 & & & 1 & 0.6 & & & 7 & 2.3 & & \\
\hline \multicolumn{13}{|l|}{ Education } \\
\hline Elementary school & 20 & 14.4 & & & 8 & 4.8 & & & 28 & 9.1 & & \\
\hline Middle school & 19 & 13.7 & & & 10 & 6.0 & & & 29 & 9.4 & & \\
\hline High school graduate & 58 & 41.7 & & & 56 & 33.3 & & & 114 & 37.1 & & \\
\hline College graduate & 33 & 23.7 & & & 82 & 48.8 & & & 115 & 37.5 & & \\
\hline No response & 9 & 6.5 & & & 12 & 7.1 & & & 21 & 6.8 & & \\
\hline \multicolumn{13}{|c|}{ Household income (dollars/month) } \\
\hline Less than $\$ 1,650$ & 72 & 51.8 & & & 46 & 27.4 & & & 118 & 38.4 & & \\
\hline$\$ 1,650-3,300$ & 33 & 23.7 & & & 57 & 33.9 & & & 90 & 29.3 & & \\
\hline$\$ 3,300-4,950$ & 13 & 9.4 & & & 31 & 18.5 & & & 44 & 14.3 & & \\
\hline More than $\$ 4,950$ & 2 & 1.4 & & & 12 & 7.1 & & & 14 & 4.6 & & \\
\hline No response & 19 & 13.7 & & & 22 & 13.1 & & & 41 & 13.4 & & \\
\hline \multicolumn{13}{|l|}{ Disease activity category } \\
\hline DAS28-CRP <2.6 & 93 & 66.9 & & & & & & & & & & \\
\hline ASDAS-CRP $<1.3$ & & & & & 75 & 44.6 & & & & & & \\
\hline \multicolumn{13}{|l|}{ Radiographic progression } \\
\hline Presence of erosive change & 76 & 54.7 & & & & & & & & & & \\
\hline Modified New York criteria & & & & & 65 & 38.7 & & & & & & \\
\hline Grade 4 sacroiliitis & & & & & & & & & & & & \\
\hline \multicolumn{13}{|l|}{ Comorbidities } \\
\hline Hypertension & 42 & 29.5 & & & 38 & 22.6 & & & 79 & 25.7 & & \\
\hline Diabetes mellitus & 18 & 12.9 & & & 9 & 5.4 & & & 27 & 8.8 & & \\
\hline Hyperlipidemia & 22 & 15.8 & & & 30 & 17.9 & & & 52 & 16.9 & & \\
\hline Osteoporosis & 39 & 28.1 & & & 6 & 3.6 & & & 45 & 14.7 & & \\
\hline Fibromyalgia & 10 & 7.2 & & & 7 & 4.2 & & & 17 & 5.5 & & \\
\hline
\end{tabular}

95\% CI: 0.62-0.82). The percentage of patients who answered satisfactory was higher among AS $(82.2 \%$ of patients and $77.4 \%$ of physicians, weighted $\kappa: 0.62,95 \%$ CI: 0.52-0.72) than RA. In both groups, satisfaction tended to be higher in patients than physicians.

The most preferred route of administration was subcutaneous (41.3\%), followed by intravenous (32.0\%), and oral (26.7\%). Most of AS patients preferred subcutaneous administration (50.0\%), while RA patients mostly preferred intravenous administration (39.3\%; Figure 3a). Most current users of intravenous administration methods preferred intravenous infusion (68.9\%), and the majority of current users of subcutaneous methods preferred subcutaneous injection (58.4\%). The most preferred location for medication administration was a clinic (52.4\%), followed by patient's home $(47.6 \%)$ in the whole population. However, the AS 


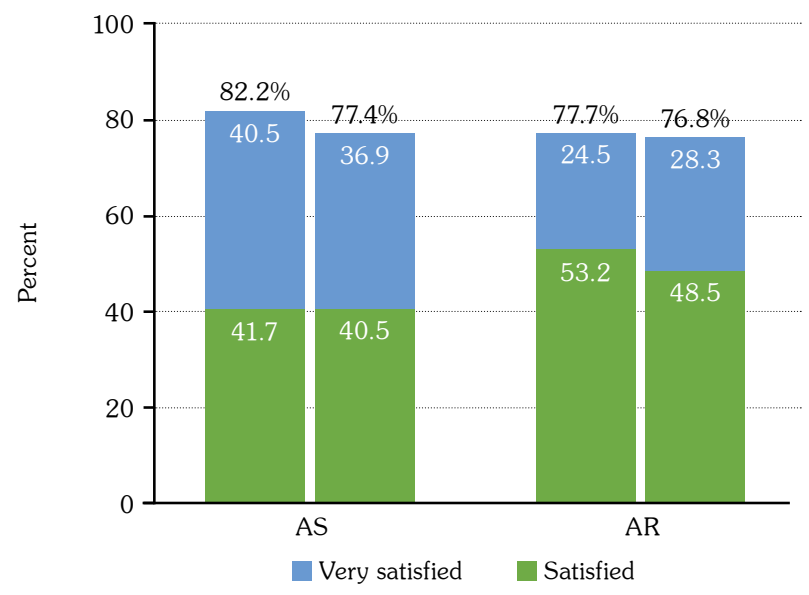

Figure 2. Percentage of patients and physicians who were satisfied or very satisfied with the current biologic (rated on a 5-point Likert-type scale).

AS: Ankylosing spondylitis; RA: Rheumatoid arthritis.

and RA patients had different answers. The AS patients preferred receiving medication at home (53.6\%), while RA patients preferred clinic-based medication administration (59.7\%; Figure 3b). These percentages roughly corresponded to the patients' current administration site: $53.3 \%$ of AS patients had their medication administered at home and $60.4 \%$ of RA patients had theirs administered at a clinic.

Only $35.2 \%$ of patients reported well and/or very well knowledge about the therapeutic effects, side effects, and administration methods of their current bDMARD (Supplementary Table 1). In addition, $86.6 \%$ of the patients cited their physician as the primary source of information about biological treatment (Supplementary Table 2). In terms of education, the patients were mostly willing to be informed about the medication's therapeutic effect (46.1\%), followed by possible adverse events (24.2\%), emergency tips (11.8\%), cost (9.4\%), and method of administration (8.4\%) by their healthcare providers (Supplementary Table 3).

The patients reported that the most important factor while considering a biologic were therapeutic effect (69.3\%), safety (12.4\%), physician's recommendation (10.1\%), cost (4.6\%), and convenience of administration (3.6\%). These proportions were similar among AS patients; however, RA patients were more likely to prioritize physician's recommendation (12.9\%) than safety (Supplementary Table 4).

The most anticipated effect of biological treatment was a decrease in pain (52.2\%), followed by prevention of the disease progression (19.8\%), improvement of inflammatory markers (18.8\%), reduction in stiffness (5.3\%), and improvement of joint function (4.0\%). The patients with AS showed similar anticipations to the answers of whole population, while RA patients were more likely to expect improvement of inflammatory markers $(24.5 \%)$ than prevention of the disease progression (21.6\%) (Supplementary Table 5).

There were $13.4 \%$ patients $(13.0 \%$ of RA and $13.8 \%$ of AS) who experienced reduction in the number of doses or discontinued medications on (a)

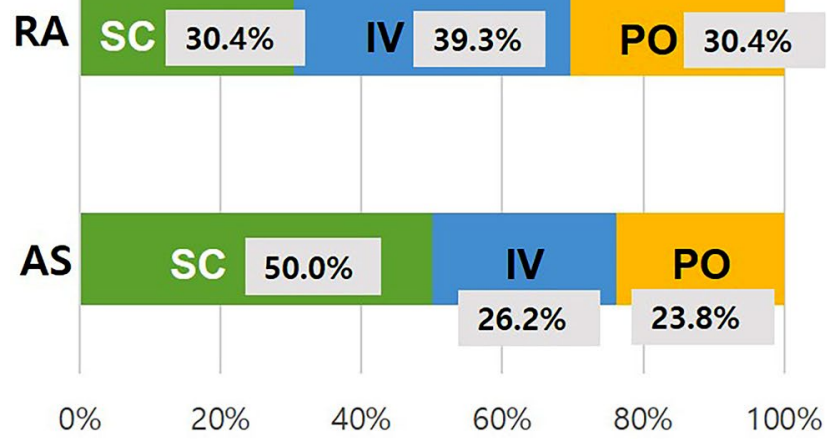

(b)

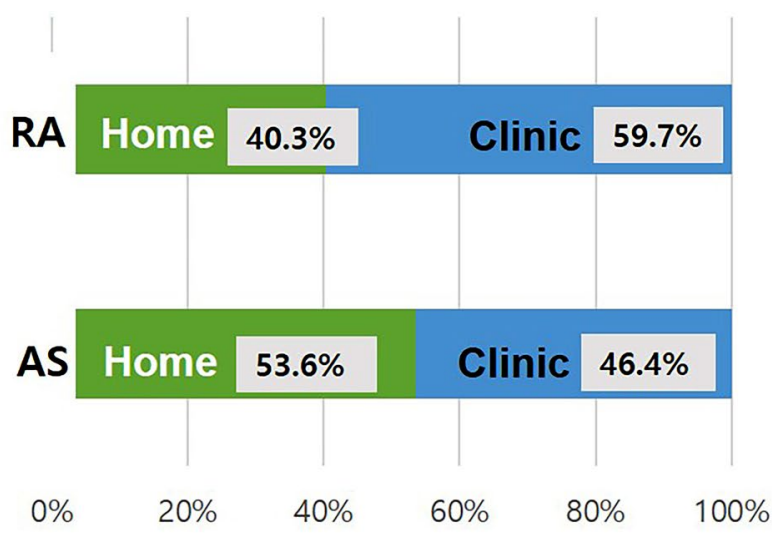

Figure 3. Patients' preferred route (a) and location (b) of medication administration. RA: Rheumatoid arthritis; SC: Subcutaneous; IV: Intravenous; PO: Per oral; AS: Ankylosing spondylitis. 
their own. There was no significant difference in physician global assessment, patient Visual Analog Scale (VAS) scores, ESR, CRP, swollen or tender joint count in RA, or back pain/morning stiffness/peripheral joint pain in AS at the time of survey between the patients with and without the experience of non-adherence.

Forty-four (14.4\%; 19 RA and 25 AS) patients reported having experienced adverse events. The most common of them included injection site reaction (5.8\%) and other skin problems (3.2\%). Other patients reported additional symptoms such as dyspnea, chest discomfort, frequent upper respiratory tract infections, fatigue, headaches, weight gain, insomnia, and facial flushing. Only one patient had a serious adverse event requiring admission. A 44-year-old female RA patient experienced anaphylactic shock after taking rituximab.

\section{DISCUSSION}

In our study, patient satisfaction with biologics was relatively high. In addition, there was a good correlation between patient and physician satisfaction, although the former tended to be higher than the latter. In a comparison of satisfaction based on diagnosis, AS patients were more likely to be satisfied than RA patients. Interestingly, the patients preferred injections to oral medications, particularly RA patients. Although the main topic of this study was bDMARD, we added the oral option to a questionnaire for the most preferred route of administration. As a synthetic targeted DMARD, such as Janus kinase inhibitor, has been emerged as an alternative to bDMARD. Our finding conflicts with those of previous studies on early RA patients, which found that RA patients mainly preferred oral medication to injection. ${ }^{6,8}$ However, our results are consistent with those of other recent studies that patients using intravenous remedies showed high satisfaction with the effectiveness of the treatment, and patients who have experienced effective intravenous biological treatment were more open to further intravenous treatment. ${ }^{5,11}$ The participants in our study were already using injectable agents, and about 70\% were using the injections for more than a year and adapted to their use. These results are limited to patients who already have biologics. Based on the results of our study and other studies, it can be inferred that early arthritis patients tend to prefer oral medications, but after experiencing treatment failure with oral medications and becoming familiar with the use of injectable agents, they are more open to the use of injectable agents. Our findings also support several previous studies reporting that subcutaneous injections are preferred among AS patients. ${ }^{12,13}$ These results may be due to preference for self-injection and their negative experiences of previous treatment, such as ineffectiveness or gastrointestinal discomforts, while taking non-steroidal anti-inflammatory drugs or sulfasalazine.

In the current study, the general, patients were more concerned with the therapeutic effect than cost or convenience while selecting a biologic, consistent with the findings of previous studies.7,14 Our findings suggest that patients can afford high costs and inconvenience to achieve good treatment outcomes. However, these findings differ from those of previous studies that patients often placed a higher importance on the cost of medical treatment from another country. ${ }^{15,16}$ This discrepancy is thought to be caused by difference in health insurance system. Almost all patients in this study received payment assistance for rare and incurable diseases from the Korean National Health Insurance Service, and paid only $10 \%$ of their total medical costs. ${ }^{17}$ Therefore, patients' perceptions of drug costs should be interpreted differently, according to a country's health insurance and social welfare systems.

Patients' understanding of the effectiveness and side effects of therapeutic agents is crucial for proper management, particularly for chronic diseases such as RA and AS. However, only $35.2 \%$ of patients who participated in our study answered that they knew about the therapeutic effects, side effects, and administration methods of their biological agents well or very well. Considering that $86.6 \%$ of patients cited their physicians as their primary source of information, physicians' education for patients seems to be insufficient. In Korea, many rheumatologists cannot spend enough time on each patient, as there are too many patients in outpatient clinics. One Korean study showed that rheumatologists in the outpatient clinics spent only $57.8 \%$ of the expected time which patients would be satisfied with. ${ }^{18}$ Physicians should spend more time for 
providing information about treatment plan and therapeutic agents to patients, and this requires improvement of the medical system, as well as efforts paid by physicians.

Drug adherence is an essential part of effective treatment, and it is important for the patients to be aware of their condition and current therapeutic agents. Beliefs and perceptions about therapeutic agents were significantly associated with non-adherence. One Korean study showed that intentional non-adherence was associated with a lower belief in the therapeutic agents and a higher emotional stress to disease in RA patients. ${ }^{19}$ When patients' knowledge and belief about their disease and therapeutic agents are lacking, it can lead to non-adherence and unsatisfactory treatment outcome. Patient education can increase adherence to medication, and make patients participate their treatment actively. ${ }^{20}$ For appropriate education, physician should know patient's expectations and concerns about the current treatment. Physician's understanding and support for patients' perceptions and concerns would improve the doctor-patient relationship and make a shared decision-making possible.

The strength of this study is that data were collected from six hospitals representing each region in South Korea, including various patients, and the questionnaire included overall perspective on bDMARD. However, there are some limitations to this study. First, disease duration and joint damage might be major confounders in satisfaction measure. Second, we did not cover all diseases of inflammatory arthritis such as psoriatic arthritis, and juvenile idiopathic arthritis. However, considering most patients with inflammatory patients receiving bDMARD in Korea are those with RA and AS, we included the majority of patients.

In conclusion, patients value therapeutic effect more than cost or convenience while selecting a bDMARD, and consider their physicians to be the primary information source. Therefore, it is important for physicians to provide appropriate education and encourage patients to cooperate actively with treatment. We believe that our study would be a guide for physicians to be aware of patient's needs and make an education plan for patients who need to use biological agents.

\section{Acknowledgments}

The authors thank Chan Hwan Seong and Hyo Jin Min, Department of Clinical Pharmacology \& Therapeutics, Chungbuk National University Hospital, for their assistance with statistical procedures for this study.

\section{Declaration of conflicting interests}

The authors declared no conflicts of interest with respect to the authorship and/or publication of this article.

\section{Funding}

The authors received no financial support for the research and/or authorship of this article.

\section{REFERENCES}

1. Selmi C, Generali E, Massarotti M, Bianchi G, Scire CA. New treatments for inflammatory rheumatic disease. Immunol Res 2014;60:277-88.

2. Fleischmann $\mathrm{R}$, Tongbram $\mathrm{V}$, van Vollenhoven $\mathrm{R}$, Tang DH, Chung J, Collier D, et al. Systematic review and network meta-analysis of the efficacy and safety of tumour necrosis factor inhibitor-methotrexate combination therapy versus triple therapy in rheumatoid arthritis. RMD Open 2017;3:e000371.

3. Smolen JS, Landewé RBM, Bijlsma JWJ, Burmester GR, Dougados M, Kerschbaumer A, et al. EULAR recommendations for the management of rheumatoid arthritis with synthetic and biological diseasemodifying antirheumatic drugs: 2019 update. Ann Rheum Dis 2020;79:685-99.

4. van der Heijde D, Ramiro S, Landewé R, Baraliakos X, Van den Bosch F, Sepriano A, et al. 2016 update of the ASAS-EULAR management recommendations for axial spondyloarthritis. Ann Rheum Dis 2017;76:978-91.

5. Grisanti L, Kwiatkowski A, Dyrda P, Field E, Grisanti $\mathrm{J}$, Hatem $\mathrm{J}$, et al. Patient Perspectives on Intravenous Biologics for Rheumatologic Disease. Arthritis Care Res (Hoboken) 2019;71:1234-42.

6. Fayad F, Ziade NR, Merheb G, Attoui S, Aiko A, Mroue $\mathrm{K}$, et al. Patient preferences for rheumatoid arthritis treatments: results from the national crosssectional LERACS study. Patient Prefer Adherence 2018;12:1619-25.

7. Cinar FI, Cinar M, Yilmaz S, Simsek I, Erdem H, Pay $\mathrm{S}$. Thoughts and perceptions of ankylosing spondylitis patients with regard to TNF inhibitors. Rheumatol Int 2014;34:979-86.

8. Hazlewood GS, Bombardier C, Tomlinson G, Thorne C, Bykerk VP, Thompson A, et al. Treatment preferences of patients with early rheumatoid arthritis: a discrete-choice experiment. Rheumatology (Oxford) 2016;55:1959-68. 
9. Prevoo ML, van 't Hof MA, Kuper HH, van Leeuwen MA, van de Putte LB, van Riel PL. Modified disease activity scores that include twenty-eight-joint counts. Development and validation in a prospective longitudinal study of patients with rheumatoid arthritis. Arthritis Rheum 1995;38:44-8.

10. Machado PM, Landewé R, Heijde DV; Assessment of SpondyloArthritis international Society (ASAS). Ankylosing Spondylitis Disease Activity Score (ASDAS): 2018 update of the nomenclature for disease activity states. Ann Rheum Dis 2018;77:1539-40.

11. Gaylis NB, Sagliani J, Black S, Tang KL, DeHoratius R, Kafka WA, et al. Patient-reported outcome assessment of inflammatory arthritis patient experience with intravenously administered biologic therapy. Patient Prefer Adherence 2017;11:1543-53.

12. Nolla JM, Rodriguez M, Martin-Mola E, Raya E, Ibero I, Nocea G, et al. Patients' and rheumatologists' preferences for the attributes of biological agents used in the treatment of rheumatic diseases in Spain. Patient Prefer Adherence 2016;10:1101-13.

13. Scalone L, Sarzi-Puttini P, Sinigaglia L, Montecucco C, Giacomelli R, Lapadula G, et al. Patients', physicians', nurses', and pharmacists' preferences on the characteristics of biologic agents used in the treatment of rheumatic diseases. Patient Prefer Adherence 2018;12:2153-68.

14. Diaz-Torne C, Urruticoechea-Arana A, Ivorra-Cortes J, Diaz S, Dilla T, Sacristan JA, et al. What Matters Most to Patients and Rheumatologists? A Discrete
Choice Experiment in Rheumatoid Arthritis. Adv Ther 2020;37:1479-95.

15. Hifinger M, Hiligsmann M, Ramiro S, Severens JL, Fautrel B, Watson V, et al. Patients' preferences and economic considerations play an important role in treatment decisions: a discrete choice experiment among rheumatologists. Rheumatology (Oxford) 2017;56:68-76.

16. Augustovski F, Beratarrechea A, Irazola V, Rubinstein F, Tesolin P, Gonzalez J, et al. Patient preferences for biologic agents in rheumatoid arthritis: a discretechoice experiment. Value Health 2013;16:385-93.

17. Choi IA, Lee JS, Song YW, Lee EY. Mortality, disability, and healthcare expenditure of patients with seropositive rheumatoid arthritis in Korea: A nationwide population-based study. PLoS One 2019;14:e0210471.

18. Hee LC, Hyunsun L, Youngnam K, Hee PA, Eun-Cheol $\mathrm{P}$, Jung-Gu K. Analysis of Appropriate Outpatient Consultation Time for Clinical Departments. Health Policy and Management 2014;24(3):254-60.

19. Suh YS, Cheon YH, Kim HO, Kim RB, Park KS, $\mathrm{Kim} \mathrm{SH}$, et al. Medication nonadherence in Korean patients with rheumatoid arthritis: the importance of belief about medication and illness perception. Korean J Intern Med 2018;33:203-10.

20. Taibanguay N, Chaiamnuay S, Asavatanabodee P, Narongroeknawin P. Effect of patient education on medication adherence of patients with rheumatoid arthritis: a randomized controlled trial. Patient Prefer Adherence 2019;13:119-29. 


\section{Supplement 1}

\section{Patient awareness survey on biologic agent}

Center ID:

Patient ID:

Occupation: Office Worker/Self Employment/Physical Labor/Housewife/Student/Unemployment/Others (......................)

Education level: Elementary school graduate/Junior school graduate/Junior high school graduate/University graduate

Monthly average income: less than 1,600 dollars/1,600 to 3,199 dollars/3,200 to 4,799 dollars/4,800 dollars or more

1. How satisfied are you with the therapeutic effectiveness of your current biological agent?

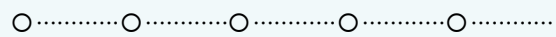

Very dissatisfied-dissatisfied---moderate---satisfied--very satisfied

If you are not satisfied with the effectiveness of treatment, what is the reason? ( ...)

2. What is the most important thing when considering biologics? (Select one)

1) Treatment effect 2) Stability 3) Convenience of medication 4) Reasonable price 5) Doctor's recommendation 6) Other (......

3. Why did you choose the biologic agent you are using? (Multiple responses possible)

1) Treatment effect 2) Stability 3) Convenience of medication 4) Reasonable price 5) Doctor's recommendation 6) Other (..... ...)

4. What are the most anticipated effects of using biologic agent? (Select one)

1) Reduction of pain 2) Improvement of stiffness 3) Improvement of inflammation level 4) Improvement of joint function

5) Suppression of disease progression

5. Among the methods of administration of biological agents, please rank which method you prefer $\left(1^{\text {st }}, 2^{\text {nd }}, 3^{\text {rd }}\right)$

Subcutaneous injection (.....

..) Intravenous injection (.

..) Oral administration (.....

6. Which place do you prefer to administer the biological agent?

1) Home 2) Clinic 3) Other (.....

....)

7. How well do you know the properties of the drugs you are using (therapeutic effects, side effects, methods of administration, etc.)? Very Unsure-Unsure-Moderate-Well Known-Very Good

8. Where do you usually get information about biologic agents? (Multiple responses possible) 1) Doctor 2) Nurse 3) Pharmacist 4) Internet 5) Patients Organization 6) Other

9. Are there any concerns about using biological agents? (Multiple responses possible)

1) Lack of effectiveness 2) Side effects 3) Expensive price 4) Uncertainty in the duration of administration 5) Other (

10. Are you familiar with the side effects of the current biological agent?

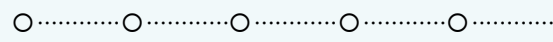

Very Unsure-Unsure-Moderate-Well Known-Very Good

11. Are there any inconveniences with using biological products?

1) Yes 2) No

What is it? (. (...)

12. Are there any inconveniences with using biological products?

1) Yes 2) No

If yes, what side effects have you had? (

If yes, what medication was it? (. ...)

13. Have you ever experienced a worsening of your existing illness (other than current rheumatic disease) while using biological agents?

1) Yes 2) No

If yes, what is it?

1) Respiratory system diseases 2) Digestive system diseases 3) Skin diseases 4) Infectious diseases 5) Allergic diseases 6) Others (.

14. Do you have difficulties in your daily life with the use of biologics?

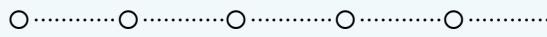

Not at all - No - Moderate - Difficult - Very Difficult

15. Have you received education on a biologic agent before using it?

1) Received 2) Not received

If received, from whom did you get? (Multiple responses possible)

1) Doctor 2) Nurse 3) Pharmacist 4) Pharmaceutical company 5) Other (. 


\section{Supplement 1. Continued}

16. Where are the biologic agent being administered? (Multiple responses possible)

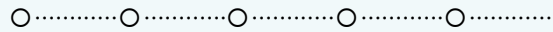

1) Your place 2) Other's house 3) Clinic 4) Other (.........

....)

How difficult is self-injection?

Not difficult at all - not difficult - moderate - difficult - very difficult

If you have difficulty in self-injection, what kind of difficulty is it? (

17. Are you familiar with the method of administration (dose and interval) of biological products?

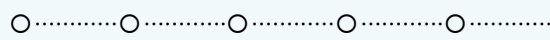

Not at all - Not - Normal - Knowing - Very familiar

18. How accurately do you keep the interval between biologics administered?

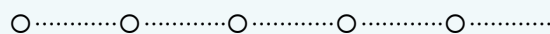

Very bad - not good - moderate - very good - very good

19. Have you ever adjusted the number of doses or stopped taking medication without consulting your doctor?

1) Yes 2) No

If so, why? (.

20. When using biologics, please rank what you expect from medical staff (1st to 5 th)

1) Instruction of administration method (.

2) Comparison of drug efficacy (...... ....)

3) Description of drug side effects (......

4) Expense description (. (...)

5) How to respond in an emergency (...

Please write anything you would like other than the ones suggested above (

Supplementary Table 1. Patients' levels of knowledge regarding current biologic agent and sources of information

\begin{tabular}{|c|c|c|c|c|c|c|}
\hline & \multicolumn{2}{|c|}{ RA } & \multicolumn{2}{|c|}{ AS } & \multicolumn{2}{|c|}{ Total } \\
\hline & $\mathrm{n}$ & $\%$ & $\mathrm{n}$ & $\%$ & $\mathrm{n}$ & $\%$ \\
\hline Know very well & 8 & 5.8 & 5 & 3.0 & 13 & 4.2 \\
\hline Know well & 27 & 19.4 & 29 & 17.3 & 56 & 18.2 \\
\hline Neutral & 61 & 43.9 & 72 & 42.9 & 133 & 43.3 \\
\hline Not really & 37 & 26.6 & 54 & 32.1 & 91 & 29.6 \\
\hline Not at all & 6 & 4.3 & 8 & 4.8 & 14 & 4.6 \\
\hline Total & 139 & 45.3 & 168 & 54.7 & 307 & 100.0 \\
\hline
\end{tabular}

Supplementary Table 2. Sources of information on biologic agents

\begin{tabular}{|c|c|c|c|c|c|c|}
\hline & \multicolumn{2}{|c|}{ RA } & \multicolumn{2}{|c|}{ AS } & \multicolumn{2}{|c|}{ Total } \\
\hline & $\mathrm{n}$ & $\%$ & $\mathrm{n}$ & $\%$ & $\mathrm{n}$ & $\%$ \\
\hline Doctor & 125 & 91.2 & 141 & 86.5 & 266 & 88.7 \\
\hline Nurse & 11 & 8.0 & 11 & 6.8 & 22 & 7.3 \\
\hline Pharmacist & 5 & 3.7 & 3 & 1.8 & 8 & 2.7 \\
\hline Internet & 20 & 14.6 & 44 & 27.0 & 64 & 21.3 \\
\hline Patient association & 5 & 3.7 & 13 & 8.0 & 18 & 6.0 \\
\hline Others & 2 & 1.5 & 6 & 3.7 & 8 & 2.7 \\
\hline Total & 137 & 45.7 & 163 & 54.3 & 300 & 100.0 \\
\hline
\end{tabular}


Supplementary Table 3. Patient education topics preferred by patients

\begin{tabular}{|c|c|c|c|c|c|c|}
\hline & \multicolumn{2}{|c|}{ RA } & \multicolumn{2}{|c|}{ AS } & \multicolumn{2}{|c|}{ Total } \\
\hline & $\mathrm{n}$ & $\%$ & $\mathrm{n}$ & $\%$ & $\mathrm{n}$ & $\%$ \\
\hline Method of administration & 12 & 8.7 & 13 & 8.2 & 25 & 8.4 \\
\hline Medication's therapeutic effect & 61 & 44.2 & 76 & 47.8 & 137 & 46.1 \\
\hline Possible adverse events & 31 & 22.5 & 41 & 25.8 & 72 & 24.2 \\
\hline Cost & 20 & 14.5 & 8 & 5.0 & 28 & 9.4 \\
\hline Emergency tips & 14 & 10.1 & 21 & 13.2 & 35 & 11.8 \\
\hline Total & 138 & 46.5 & 159 & 53.5 & 297 & 100.0 \\
\hline
\end{tabular}

Supplementary Table 4. Key considerations when choosing a biologic agent

\begin{tabular}{|c|c|c|c|c|c|c|}
\hline & \multicolumn{2}{|c|}{ RA } & \multicolumn{2}{|c|}{ AS } & \multicolumn{2}{|c|}{ Total } \\
\hline & $\mathrm{n}$ & $\%$ & $\mathrm{n}$ & $\%$ & $\mathrm{n}$ & $\%$ \\
\hline Effectiveness & 96 & 69.6 & 116 & 69.1 & 212 & 69.3 \\
\hline Safety & 11 & 8.0 & 27 & 16.1 & 38 & 12.4 \\
\hline Convenience of administration & 6 & 4.4 & 5 & 3.0 & 11 & 3.6 \\
\hline Cost & 7 & 5.1 & 7 & 4.2 & 14 & 4.6 \\
\hline Physician's recommendation & 18 & 13.0 & 13 & 7.7 & 31 & 10.1 \\
\hline Total & 138 & 45.1 & 168 & 54.9 & 306 & 100.0 \\
\hline
\end{tabular}

Supplementary Table 5. Anticipated effects of biologic treatment

\begin{tabular}{|c|c|c|c|c|c|c|}
\hline & \multicolumn{2}{|c|}{ RA } & \multicolumn{2}{|c|}{ AS } & \multicolumn{2}{|c|}{ Total } \\
\hline & $\mathrm{n}$ & $\%$ & $\mathrm{n}$ & $\%$ & $\mathrm{n}$ & $\%$ \\
\hline Decrease in pain & 63 & 45.3 & 95 & 57.9 & 158 & 52.2 \\
\hline Improvement of stiffness & 5 & 3.6 & 11 & 6.7 & 16 & 5.3 \\
\hline Improvement of inflammatory markers & 34 & 24.5 & 23 & 14.0 & 57 & 18.8 \\
\hline Improvement of joint function & 7 & 5.0 & 5 & 3.1 & 12 & 4.0 \\
\hline Prevention of the disease's progression & 30 & 21.6 & 30 & 18.3 & 60 & 19.8 \\
\hline Total & 139 & 45.9 & 164 & 54.1 & 303 & 100.0 \\
\hline
\end{tabular}

